\title{
Is CABG the preferred method of revascularization in multivessel coronary artery disease?
}

Hannan EL, Racz MJ, Walford G, Jones RH, Ryan TJ, Bennet E, et al. Long-term outcomes of coronary-artery bypass grafting versus stent implantation. N Engl J Med 2005;352(21):2174-83.

Background: Clinicians still debate the merits of percutaneous coronary intervention (PCI) relative to coronary artery bypass grafting (CABG) for patients with multivessel coronary artery disease. To date, only 4 randomized clinical trials have compared the outcomes of PCI and stent implantation with those of CABG. ${ }^{1,2}$ Such studies have been too highly selective, small and underpowered to demonstrate a consistent and compelling mortality advantage of one modality over the other.

Design: This retrospective study compared outcomes for 22102 patients undergoing PCI and stenting with 37212 patients undergoing CABG between Jan. 1, 1997, and Dec. 30, 2000. Primary outcomes included mortality and subsequent revascularization within 3 years. Patients were further categorized into 5 anatomic subgroups depending on their number of diseased vessels and presence or absence of disease involving the proximal left anterior descending coronary artery (LAD). Mortality analyses were adjusted for disease severity through use of a multivariate Cox proportional hazard model.

Results: Compared with patients who underwent PCI, those who underwent CABG were older and at baseline were more likely to have disease affecting 3 vessels, a lower ejection fraction and a high prevalence of comorbidities including cerebrovascular and peripheral vascular disease, diabetes and renal failure. Subsequent rates of revascularization were significantly lower among patients who received $\mathrm{CABG}$ rather than stenting. $\mathrm{Al}$ though the in-hospital mortality rate was significantly higher in the CABG group than in the stenting group $(1.75 \%$ v. $0.68 \%$, $p<0.001)$, the converse was true for long-term mortality. Specifically, the adjusted 3-year mortality rates were lower among CABG patients, regardless of their anatomic distribution of disease, existence of diabetes or impairment of left ventricular function. The survival benefits associated with CABG were more pronounced and consistent among patients with 3 -vessel disease and involvement of the proximal LAD (adjusted hazard ratio [HR] 0.64, 95\% confidence interval [CI] 0.56-0.74) than among those with 2-vessel disease and nonproximal LAD involvement (adjusted HR 0.76, 95\% CI 0.60-0.96; Table 1).

Commentary: The advantages of this study included its large sample, clinical detail and ability to adjust for differences in clinical and anatomic character- istics. Moreover, the advantage associated with CABG persisted despite the fact that these patients were modestly older and had more comorbidities at baseline than those receiving PCI. The outcome benefits observed to be associated with CABG rather than PCI were also biologically plausible and consistent with the findings of at least 1 meta-analysis, ${ }^{1}$ which combined stented and nonstented patients. The study has $2 \mathrm{im}$ portant limitations: First, its observational design would not have adequately accounted for unmeasured confounding factors. Second, these findings may not be generalizable to the Canadian health care system, where delays before such interventions may occur. ${ }^{3}$ Moreover, the technology for both treatments is rapidly changing, most notably with the introduction of drug-eluting stents.

Practice implications: These results suggest that long-term outcomes in patients with multivessel disease may be superior with CABG than with stent implantation. Nonetheless, physicians must still consider multiple factors when choosing a revascularization modality. First, the long-term survival benefit associated with CABG must be weighed against the higher incremental short-term (in-hospital) risks of complications and death related to the procedure. Accordingly, such decision-making must take into

Table 1: Hazard ratios* for death after CABG compared with those after stenting

\begin{tabular}{|c|c|c|c|c|c|c|}
\hline \multirow[b]{2}{*}{ Disease location } & \multicolumn{2}{|c|}{2 -vessel disease, $n$} & \multirow{2}{*}{$\begin{array}{c}\text { Hazard ratio } \\
(95 \% \mathrm{Cl})\end{array}$} & \multicolumn{2}{|c|}{3 -vessel disease, $n$} & \multirow{2}{*}{$\begin{array}{c}\text { Hazard ratio } \\
(95 \% \mathrm{Cl})\end{array}$} \\
\hline & Stent & CABG & & Stent & CABG & \\
\hline No LAD disease & 5847 & 1309 & $0.75(0.58-0.98)$ & - & - & - \\
\hline Proximal LAD & 6033 & 8410 & $0.75(0.66-0.86)$ & 2165 & 20857 & $0.64(0.56-0.74)$ \\
\hline Nonproximal LAD & 5891 & 1690 & $0.76(0.60-0.96)$ & 2166 & 4946 & $0.74(0.62-0.90)$ \\
\hline
\end{tabular}

Note: $\mathrm{CABG}=$ coronary artery bypass grafting, $\mathrm{Cl}=$ confidence interval, $\mathrm{LAD}=$ left anterior descending coronary artery. *Adjusted for age, sex, ejection fraction, and presence or absence of diabetes, congestive heart failure, chronic obstructive pulmonary disease, carotid artery disease, aortoiliac disease, shock, renal failure, femoral or popliteal disease, and stroke. 
account patient preferences, competing risks and natural lifeexpectancy. Second, interregional and interhospital variations in procedural availability may circumvent or mitigate adverse consequences that may be associated with service delays. In short, decisions about which revascularization procedure to use still require sound clinical judgement in the context of evidence-based medicine.
Harindra C. Wijeysundera

\section{David A. Alter}

Division of Cardiology

Sunnybrook and Women's College

Health Sciences Centre

Institute for Clinical Evaluative

Sciences

University of Toronto

Toronto, Ont.

\section{References}

1. Hoffman SN, TenBrook JA, Wolf MP, Pauker SG, Salem DN, Wong JB. A meta-analysis of randomized controlled trials comparing coronary artery bypass graft with percutaneous transluminal coronary angioplasty: one- to eight-year outcomes. $7 \mathrm{Am}$ Coll Cardiol 2003;41(8):1293-304.

2. SoS Investigators. Coronary artery bypass surgery versus percutaneous coronary intervention with stent implantation in patients with multivessel coronary artery disease (the Stent or Surgery trial): a randomized controlled trial. Lancet 2002;360(9338):965-70.

3. McGlynn EA, Naylor CD, Anderson GM, Leape LL, Park RE, Hilborne $\mathrm{LH}$, et al. Comparison of the appropriateness of coronary angiography and coronary artery bypass graft surgery between Canada and New York State. FAMA 1994;272(12):934-40.

\title{
A CMAJ Call for Medical Images: Clinical Vistas
}

\section{Send us your interesting clinical images!}

Through scopes and scanners, on film and computer screens, with ultrasonography and microscopy, clinicians capture stunning images of illness and healing. CMAJ invites you to share your normally privy visual perspectives on anatomy, pathology, diagnostic procedures and therapeutic techniques. Let colleagues outside your specialty take a close look at the characteristic signs of rare conditions (Kayser-Fleischer rings in Wilson's disease) or the interior marvels of your clinical terrain (colonoscopic view of an adenomatous polyp). We're also interested in images that take a wider angle on the context of care (a recently cord-clamped newborn on a cold steel scale). If you have original, unpublished images that are beautiful or informative, rare or classic, wed like to include them in $C M A \rho_{s}$ Clinical Vistas. Be sure to include a case summary and discussion (maximum 500 words and 4 references) and a signed consent for publication from the patient (see cmaj.ca for more instructions). Video vistas are also welcome. (See CMAJ 2004;170[13]:1931 for details.)

\section{Send your images videos, or queries to:}

\section{CMAJJIAMC}

\author{
Dr. Eric Wooltorton - Canadian Medical Association Journal \\ 1867 Alta Vista Drive • Ottawa ON K1G 3 Y6 Canada \\ or pubs@cma.ca
}

